\title{
Cerebral Lupus and Cryptococcal Meningitis in a Pregnant Woman
}

\author{
Ching Soong Khoo ${ }^{1}$ Marsilla Mariaty Marzukie ${ }^{1}$ Soo Shu Yap ${ }^{2}$ Wan Nur Nafisah Wan Yahya ${ }^{1}$ \\ Hui Jan $\operatorname{Tan}^{1}$
}

${ }^{1}$ Neurology Unit, Department of Medicine, Universiti Kebangsaan
Malaysia Medical Centre, Kuala Lumpur, Malaysia
2Department of Radiology, Universiti Kebangsaan Malaysia Medical
Centre, Kuala Lumpur, Malaysia

J Neurosci Rural Pract 2020;11:183-186

\begin{abstract}
Address for correspondence Ching Soong Khoo, MRCP, Neurology Unit, Department of Medicine, Universiti Kebangsaan Malaysia Medical Centre, Jalan Yaacob Latif, Bandar Tun Razak, 56000 Cheras, Kuala Lumpur, Malaysia (e-mail: chingsoongkhoo@gmail.com).
\end{abstract}
Abstract
Keywords
- systemic lupus erythematosus
- cerebral lupus
- cryptococcal meningitis
- pregnancy

Systemic lupus erythematosus (SLE) is a chronic autoimmune and multisystem disorder, which frequently affects young women. During pregnancy, SLE flares could occur up to $65 \%$, with renal and hematological manifestations being the most common. However, reports on neuropsychiatric lupus in pregnant women are scarce. We herein report a 26-year-old lupus pregnant woman, who had cerebral lupus with concurrent cryptococcal meningitis. This case highlights the complexity in diagnosing and managing our patient to achieve the best outcome for both the mother and infant.

\section{Introduction}

Pregnant women with systemic lupus erythematosus (SLE) carry a higher maternal and fetal risk. ${ }^{1}$ Lupus mothers are at risk for pre-eclampsia and flare-ups, while their fetuses may have complications such as fetal death, premature birth, intrauterine growth restriction, and neonatal lupus syndrome. ${ }^{1}$ The rates of pregnancy loss have reduced from 43 to $17 \%$ in patients with SLE in the past four decades. ${ }^{2}$ Patients with lupus need to be stabilized at least 6 months prior to conception to reduce the risks of flare-ups. SLE tends to flare during pregnancy especially in the third trimester. ${ }^{3}$ Renal and hematologic complications are by far the commonest, while neuropsychiatric manifestations in pregnancy were reported at $3.5 \%{ }^{3,4}$

Cryptococcal meningitis (CM) is one of the more common central nervous system (CNS) infections, which occurred at 30.4 to $58.8 \%$ in SLE patients. ${ }^{5}$ Mortality rate was reported at $41.2 \%$ despite treatment. ${ }^{6}$ We describe a complex case of a pregnant woman, who had cerebral lupus with concurrent $\mathrm{CM}$ and achieved a favorable maternal and fetal outcome.

\section{Case Presentation}

A 26-year-old primigravida at 30 weeks of gestation presented with a 5-day history of headache, photophobia, fever, and vomiting. She was diagnosed with SLE, for which she had been in remission for the past 3 years. Apart from a temperature of $38^{\circ} \mathrm{C}$, systemic review was unremarkable. She was empirically treated for acute meningitis with ceftriaxone and acyclovir, as she declined lumbar puncture due to her cultural belief. On day 5 of treatment, she was discharged at own risk and did not turn up for clinic appointment.

A month later, she presented with worsening of headache, blurry vision along with her previous symptoms. In addition, she had bizarre behavior and believed that she had been possessed by evil spirits. She was orientated to person and place, but not to time, and responded to questions slowly. She had bilateral papilledema and mild neck stiffness. There was no focal neurological deficit. Her minimental state examination score was 26/30. Investigations revealed lymphopenia, elevated erythrocyte sedimentation rate (ESR), and low complements 3 and 4. Magnetic resonance imaging (MRI) of the brain revealed multiple hyperintense signals at bilateral basal ganglia ( - Fig. 1).

She refused lumbar puncture again despite multiple sessions of counseling. Taking into account all her investigation results, she was pulsed for cerebral lupus and treated for meningitis. She became more orientated, and her headaches and fever resolved. Her visual evoked potential test was normal. 

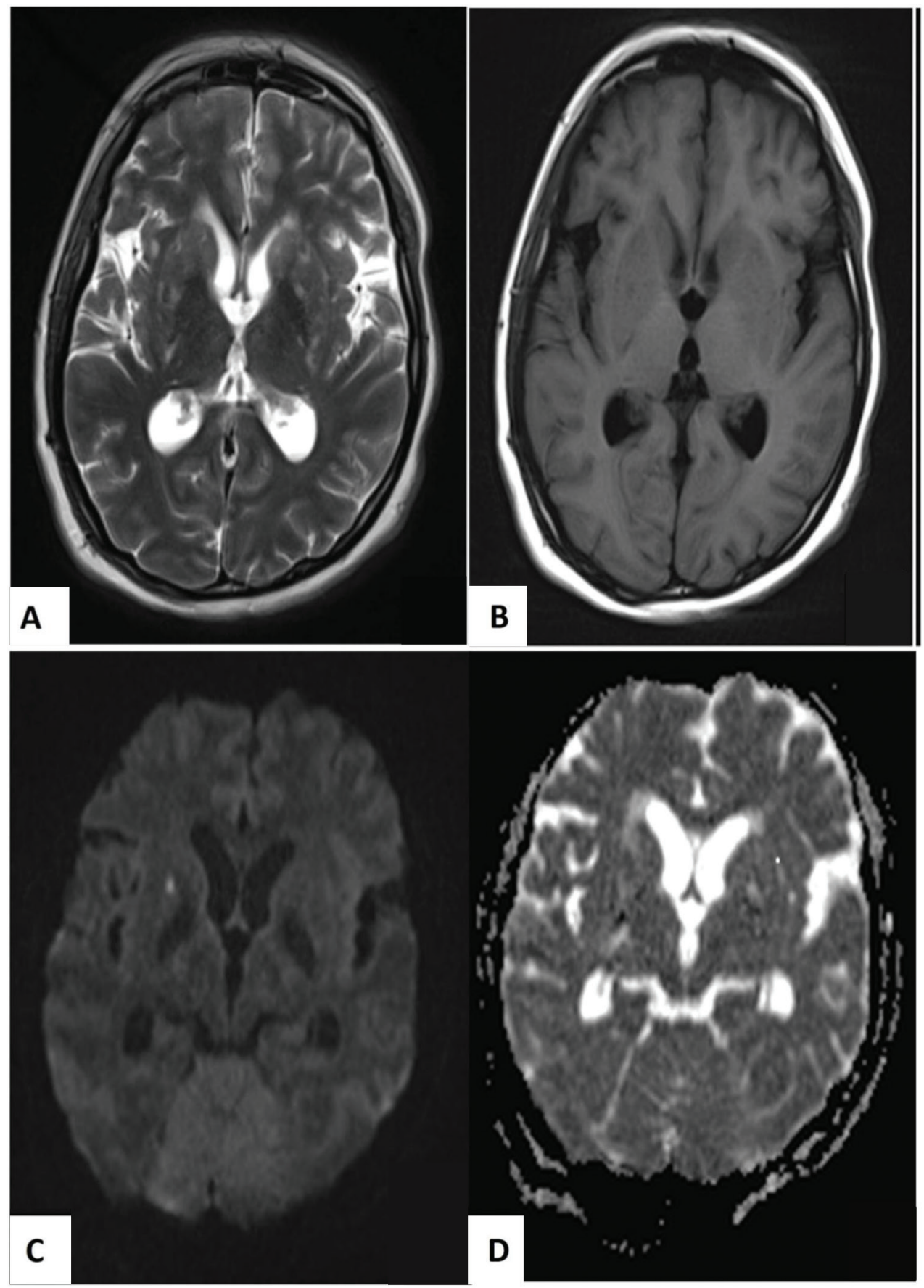

Fig. 1 Magnetic resonance imaging of the brain showed symmetrical hazy hyperintense signals in T2-weighted imaging (A) and hypointense in T1-weighted imaging (B) on bilateral basal ganglia, giving rise to "hazy brain base" sign. Acute lacunar infarcts were seen in these areas, which were hyperintense in diffusion-weighted imaging (C) and hypointense in apparent diffusion coefficient sequences (D).

After our multidisciplinary discussion, she delivered a healthy male neonate with no signs of neonatal lupus via cesarean section. However, she had a focal seizure 3 days later after delivery with high temperature. She had bilateral partial sixth cranial nerve palsy.

A repeat MRI of the brain showed the presence of communicating hydrocephalus and diffuse leptomeningeal enhancement. Magnetic resonance angiogram revealed an area of stenosis at the left posterior communicating artery (PCA) indicating the presence of vasculitis ( - Fig. 2 ). The orbits were unremarkable. She finally agreed to lumbar puncture. The opening pressure was $+43 \mathrm{CmH}_{2} \mathrm{O}$. Cryptococcal antigen was detected from the cerebrospinal fluid (CSF) at a titer of 1 : 2,560. Her CSF grew Cryptococcus neoformans.

She was immediately commenced on amphotericin B and flucytosine. She was counseled for ventriculoperitoneal shunt; however, she refused. In the ward, she required a few sessions of lumbar puncture for symptomatic headache relief. She received antifungal therapy for 30 days in the ward, with CSF culture clearance after day 24 of 


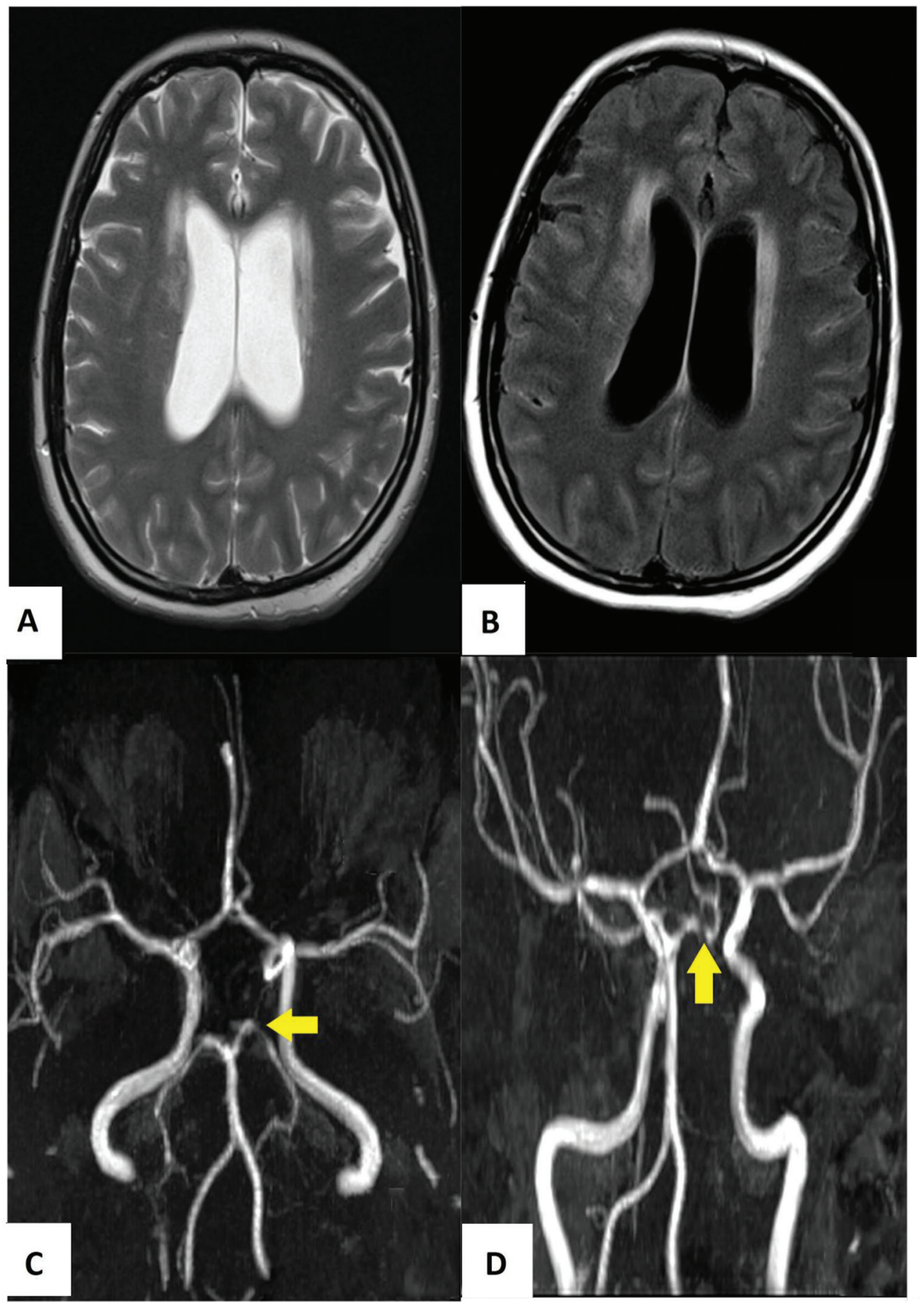

Fig. 2 The subsequent magnetic resonance imaging showed progressive hydrocephalus in T2-weighted imaging (A), while the contrast enhanced fluid-attenuated inversion recovery imaging (B) showed diffuse leptomeningeal enhancement. The time-of-flight magnetic resonance angiogram of the circle of Willis revealed a short segment stenosis in the left posterior cerebral artery (as shown by yellow arrow head) (C, D).

treatment. She was planned for a total of 6 weeks of intravenous antifungal; however, she insisted on discharge. Her medications were changed to oral fluconazole $800 \mathrm{mg}$ daily for 8 weeks for consolidation phase. In terms of cerebral lupus, she was discharged with oral prednisolone at $0.5 \mathrm{mg} / \mathrm{kg} / \mathrm{d}$.

Upon next review after completing consolidation phase, she felt better and her vision improved. She remained well after completing fluconazole $200 \mathrm{mg} / \mathrm{d}$ (maintenance phase) for 1 year.

\section{Discussion}

$\mathrm{CM}$ is an opportunistic fungal infection, with an estimated annual prevalence of $0.5 \%$ among the SLE patients. ${ }^{5}$ It was reported as the number one cause of mortality among the invasive fungal infections in the SLE population. ${ }^{7}$ However, data on CM in pregnant SLE patients are very limited.

CNS symptoms of CM are rather similar to neuropsychiatric manifestation of SLE, which explains why CM is often missed and diagnosed as cerebral lupus. Misdiagnosis leads 
one to administer larger doses of steroids or immunosuppressants for flares, which will result in catastrophic complications. A retrospective study performed by Gonzalez-Duarte et al over 2 years in eight human immunodeficiency virus (HIV)-negative patients with SLE and confirmed CM found that this group of patients had heightened immunologic activity, which rendered diagnosing CM in them difficult. ${ }^{8}$ In our case, diagnosis of cerebral lupus was confirmed with radiological evidence of vasculitic changes in the left PCA, blood parameters (low complement levels, lymphopenia, and elevated ESR), and good response to intravenous steroid. Diagnosis of concurrent CM was delayed due to the patient's refusal for lumbar puncture.

Outcome of CM in the SLE individuals is unfavorable. Death rates could go up to $50 \%$ despite antifungal therapy. ${ }^{9}$ It was demonstrated that exposure to corticosteroid $20 \mathrm{mg} / \mathrm{d}$ or its equivalent in SLE patients would predispose them to infections due to impaired cellular immunity. ${ }^{8}$

According to the Infectious Diseases Society of America, treatment in CM for non-HIV infected, nontransplant patients includes an induction phase with amphotericin B plus flucytosine for at least 4 weeks. In individuals with neurological complications such as in our case, in which she had seizures, induction therapy should be extended for 6 weeks. The treatment is then followed by a consolidation phase with fluconazole $400 \mathrm{mg}$ once daily for 8 weeks and a maintenance phase with daily fluconazole $200 \mathrm{mg}$ for 6 to 12 months. ${ }^{10}$ Flucytosine is a category $\mathrm{C}$ drug for pregnancy; hence, its use must be balanced between benefits and risks.

Another aspect to be considered in our patient is lifelong prophylactic treatment after completing the maintenance phase. Most experts recommend lifelong treatment if the patients have significant ongoing immunosuppression or persistent disease. This is also partly because of a higher relapse rate of $\mathrm{CM}$ in up to $20 \%$ of patients without maintenance therapy. ${ }^{11}$

In conclusion, a presumptive diagnosis of neuropsychiatric lupus should not preclude physicians from investigating for other differential diagnoses such as opportunistic CNS infections, in particular, CM in lupus patients to avoid delay in administering the appropriate treatment.

\section{Key Messages}

Though rare, the need to look for concomitant CNS infections such as CM in cerebral lupus individuals is important to avoid treatment delay.

\section{Funding}

None.

\section{Conflict of Interest}

None declared.

\section{References}

1 Lateef A, Petri M. Managing lupus patients during pregnancy. Best Pract Res Clin Rheumatol 2013;27(3):435-447

2 Clark CA, Spitzer KA, Laskin CA. Decrease in pregnancy loss rates in patients with systemic lupus erythematosus over a 40-year period. J Rheumatol 2005;32(9):1709-1712

3 Iozza I, Cianci S, Di Natale A, et al. Update on systemic lupus erythematosus pregnancy. J Prenat Med 2010;4(4):67-73

4 Georgiou PE, Politi EN, Katsimbri P, Sakka V, Drosos AA. Outcome of lupus pregnancy: a controlled study. Rheumatology (Oxford) 2000;39(9):1014-1019

5 Fang W, Chen M, Liu J, et al. Cryptococcal meningitis in systemic lupus erythematosus patients: pooled analysis and systematic review. Emerg Microbes Infect 2016;5(9):e95

6 Hung JJ, Ou LS, Lee WI, Huang JL. Central nervous system infections in patients with systemic lupus erythematosus. J Rheumatol 2005;32(1):40-43

7 Chen HS, Tsai WP, Leu HS, Ho HH, Liou LB. Invasive fungal infection in systemic lupus erythematosus: an analysis of 15 cases and a literature review. Rheumatology (Oxford) 2007;46(3):539-544

8 Gonzalez-Duarte A, Saniger-Alba MdelM, Higuera-Calleja J. Cryptococcal meningitis in HIV-negative patients with systemic connective tissue diseases. Neurol Res 2015;37(4):283-287

9 Matsumura M, Kawamura R, Inoue R, Yamada K, Kawano $\mathrm{M}$, Yamagishi M. Concurrent presentation of cryptococcal meningoencephalitis and systemic lupus erythematosus. Mod Rheumatol 2011;21(3):305-308

10 Perfect JR, Dismukes WE, Dromer F, et al. Clinical practice guidelines for the management of cryptococcal disease: 2010 update by the Infectious Diseases Society of America. Clin Infect Dis 2010;50(3):291-322

11 Khairullah S, Sulaiman H, Yahya F, et al. Cryptococcal meningitis and SLE: a diagnostic and therapeutic challenge. Acta Reumatol Port 2014;39(3):254-258 